\title{
The application of bilateral ultrasound phacoemulsification of cataract with IOL implantation in patients with severe somatic and psychiatric pathology
}

\begin{abstract}
Two cases of bilateral cataract removal in patients with severe somatic pathology and deep congenital psychiatric pathology are described. Patient V., with reduced vision on both eyes, suffering from multiple sclerosis with concomitant cardiovascular pathology, moving in a wheelchair had not been able to repeatedly be on inpatient treatment. Given emotional instability, the patient's reluctance to undergo another surgical intervention for a short period of time, a high risk of repeated anesthesia it was performed cataract extraction on both eyes. Visioninbothcaseswasrestored.

To patient H., with mental pathology, instillation of water-soluble fluorescein was carried out, and also hard contact lenses and a light source with a blue filter were used to determine the curvature of the cornea and the calculation of the IOL, after giving general anesthesia with artificial ventilation. Due to the complicated deformation of the trachea, intubation was impossible, and a device called "Combitube" was used.

As a result of operations, a patient with a mental pathology had vision and an orientation in the space also appeared, aggressive behavior disappeared, in the patient with severe somatic pathology the most corrected visual acuity was $0.7-0.8$.

This clinical experience shows a successful way of solving the problems posed to the ophthalmic surgeon for improving visual functions and improving the quality of life of patients with severe psychosomatic pathology. The expediency of conducting simultaneous operations on both eyes in psychic and physically restricted patients was confirmed. Also, this case allows us to recommend the Combitube device as an effective means of providing airway patency in the most difficult situations and various deformations of the trachea. This clinical experience shows a successful way of solving the problems posed to the ophthalmic surgeon for improving visual functions and improving the quality of life of patients with severe psychosomatic pathology. The expediency of conducting simultaneous operations on both eyes in psychic and physically restricted patients was confirmed. Also, this case allows us to recommend the Combitube device as an effective means of providing airway patency in the most difficult situations and various deformations of the trachea.
\end{abstract}

Keywords: cataract, bilateral phacoemulsification, IOL, imbecility, multiple sclerosis, anesthesia, mentally ill, intubation, trachea, combitube
Volume 8 Issue 3 - 2018

\section{Kliuiev GO, Luhova OM}

Health Technology Ltd, Ukraine

Correspondence: Kliuiev GO, Health Technology Ltd, Ukraine, Emailgkluev@kluev.com.ua

Received: March 28, 2018 | Published: May 15, 2018

\section{Introduction}

One of the most common causes of blindness and low vision is clouding of the lens, after achiving 40 years most of them are bilateral cataracts. When choosing the method of surgical treatment and the timing of extraction of cataracts on the second eye, ultrasonic phacoemulsification with a time interval between operations from 1 week to 1 month or more is traditionally preferred, which is considered safe in connection with the completion of postoperative reparative processes. ${ }^{1,2}$

However, with such tactics of treatment, the patient is forced to undergo a preoperative examination twice, enter the operating room twice, and receive an anesthesia twice. Also, the period of postoperative observation of the patient is doubled. Considering the fact that patients of elderly age are most often operated, this imposes additional stress on the patient's relatives, who ensure his delivery to medical institutions for operations and postoperative checks. This is especially pronounced when it is necessary to carry out operations in patients with severe somatic and mental pathology.

The aim of research is to evaluate the effectiveness of bilateral single-stage extraction of bilateral cataract in patients with severe somatic and psychiatric pathology.

\section{Materials and methods}

Two patients were observed, with a diagnosis of complicated bilateral cataract. In addition to cataract, the patients suffered from 
concomitant severe somatic and mental pathology. Given the severe concomitant somatic and mental pathology of patients, bilateral one-stage ultrasound phacoemulsification of cataracts with IOL implantation was selected as surgical treatment.

Patient V., born in 1957, suffering from multiple sclerosis with complete paralysis, complained of a decrease in vision of the both eyes for several years. During the examination, a mature, complicated cataract of the eye was identified, Vis $\mathrm{OU}=0.05$. In connection with the expressed clinical manifestations of multiple sclerosis and complete paralysis, the patient's relatives had great problems with her delivery from home to the hospital and back. It was also undesirable to undergo premedication twice, taking into account multiple sclerosis. Therefore, a decision was made on bilateral cataract extraction in both eyes. The patient was subjected to ultrasonic phacoemulsification of cataracts with the implantation of the posterior chamber IOL of SA60FT model. Operative intervention and the postoperative period passed without complications. The next day, vision in the distance was $\mathrm{OD}=0.3 ; \mathrm{OS}=0.5$, after a week $\mathrm{OD}=0.4 \mathrm{sph}-1.0=0.7 ; \mathrm{OS}=0.6$ sph-1.0 $=0.8$, near patient read the text number $5 .{ }^{1,3}$

Patient H., born in 1966, a disabled child in mental illness with complaints of a gradual decrease in the vision of both eyes, a poor orientation in space for several years. In addition, the patient suffered from imbecility and dumbness from childhood.

At visual inspection, a mature bilateral cataract was found. Preoperative diagnostics in the form of visual acuity check, keratometry and ultrasound remote echobiometry with IOL calculation was impossible due to the aggressive and unbalanced behavior of the patient. Given the difficulties in transporting and organizing the operation, blindly mute condition, aggressiveness, mental disorder of the patient, it was decided to perform ultrasound phacoemulsification of the cataract with implantation of the IOL during one surgical intervention in both eyes. It was decided to calculate the IOL after the patient was anesthetized with muscle relaxants on the operating table, just before the operation. In connection with the absence of a portable keratometer, the definition of the curvature of the cornea was carried out using a set of hard contact lenses with different radius of curvature, an aqueous solution of fluorescein and a light source with a blue filter followed by ultrasonic echo biometry.

There were performed the instillation of a water-soluble fluorescein solution and the hard contact lenses was applied, with the following light source with a blue filter. Filling by the dye the space under the lens, mainly on the periphery, indicated a "flat" landing and in the center-a "steep" landing of the HCL. In the normal position of the lens on the eye, fluorescein was evenly distributed along the under lenses space. Taking into account the basic curvature of the selected lens, the radius of the cornea was determined from the table, after which these data were used to calculate the IOL.

During giving the patient anesthesia, the anesthesia team found curvature and narrowing of the larynx and trachea, which did not allow the introduction of even a children's endotracheal tube. On attempting the intubation and direct laryngoscopy was revealed that the glottis is absolutely in accessible for detection, and all attempts to intubate blindly were unsuccessful, since the patient had an unusual deformation of the trachea.

A simple and very effective means of solving this problem was a device called "Combitube" (laryngeal obturator tube). The introduction of this device does not require the detection of a glottis, and it is simply inserted into the mouth before combining the special mark with the level of the incisors. Simultaneous cimbination of two cuffs: one at the entrance to the esophagus, the second in the oral cavity, seal the space above the vocal slit and makes breathing effective and safe. "Combitube" fully met expectations and the anesthesia passed without any complications. ${ }^{4-8}$

After entering the patient into anesthesia, it was determined the radius of curvature of the cornea with following echobiometry and calculation of the IOL on the Sonomed EZ Scan AB 5500+, and then surgical intervention.

The next day, when examined, the patient showed uniform vision, an orientation appeared in space, and aggressive behavior disappeared. At subsequent observation of the patient, vision was preserved.

In both cases the Sovereign Compact was used, intraoperative prophylaxis of infectious and inflammatory complications was performed in the form of intracameral administration of cefuroxime antibiotic and postoperative instillation of corticosteroids within 1 month in a decreasing scheme. ${ }^{9,10-13}$

\section{Results and Discussions}

After bilateral ultrasound phacoemulsification of cataract with IOL implantation in patients with severe somatic and mental pathology, the early postoperative period passed without complications. In the patient V., the maximum corrected visual acuity was $0.7-0.8$. Patient $\mathrm{H}$. appeared vision, an orientation appeared in space, and aggressive behavior disappeared. The patient recognized the mother and gestured with signs to the doctors. With subsequent monitoring of patients, vision was preserved.

The terms of inpatient stay and outpatient observation of patients have been reduced, and a high risk of repeated anesthesia has been prevented. Also, the financial and time costs of relatives were reduced, compared with the alternate eye surgery.

\section{Conclusion}

The analysis of these cases makes it possible to recommend in aggressive psychiatric patients with bilateral cataract the following:

1. Phacoemulsification of cataract with IOL implantation is expedient to be carried out simultaneously at two eyes.

2. Calculation of the IOL and the subsequent operation is performed under endotracheal anesthesia with muscle relaxants.

3. In the absence of a portable keratometer, it is possible to determine the radius of curvature of the cornea by using a test set of hard contact lenses and a fluorescein test.

4. Considering the possible concomitant pathology and intubation difficulties, in such patients, it is advisable for the anesthetist to have in his arsenal a laryngeal tube "Combitube", as an effective means of providing airway patency in the most difficult situations and various deformations of the trachea.

5. Bilateral simultaneous ultrasound phacoemulsification of cataract is justified and expedient in patients with severe somatic and mental pathology.

\section{Acknowledgments}

\section{None.}




\section{Conflict of interest}

The author declares there is no conflict of interest.

\section{References}

1. Masket S. Cataract surgical problem. Journal of catract and refractive surgery. 1997;23(10):1437-1442.

2. American Academy of Ophthalmology. Cataract in the adult eye. Preferred practice pattern. San Francisco: AAO, 2001.

3. Benezra D, Chirambo MC. Bilateral versus unilateral cataract extraction: advantages and complications. Br J Ophthalmol. 1978;62(11):770-773.

4. Beatty S, Aggarwal RK, David DB, et al. Simultaneous bilateral cataract extraction in the UK. Br J Ophthalmol.1995;79(12):1111-4.

5. Diaper CJM, Beirouty ZAY, Saba SN. Simultaneous bilateral phacoemulsification. Eur J Implant Refract Surg. 1995;7(4):232-5.

6. Ramsay AL, Diaper CJM, Saba SN, et al. Simultaneous bilateral cataract extraction. J Cataract Refract Surg. 1999;25:753-62.
7. Potamitis T, Beatty S, Pereira AM. Phacoemulsification versus endocapsular cataract extraction in a unique cohort of patients. Eye. 1996;10:551-4.

8. Arshinoff S. Simultaneous Bilateral Cataract Surgery. J Cataract Refract Surg. 1998;24(8):1015-16.

9. Covert DJ, Henry CR, Koenig SB. Intraocular lens power selection in the second eye of patients undergoing bilateral, sequential cataract extraction. Ophthalmology. 2010;117(1):1:49-54.

10. Olsen T. Use of fellow eye data in the calculation of intraocular lens power for the second eye. Ophthalmology. 2011;118(9):1710-5.

11. Sharma TK, Worstmann T. Simultaneous bilateral cataract extraction. $J$ Cataract Refract Surg. 2001;27(5):741-4.

12. Konkanen M, Kaipianinen S. Simulataneous bilateral cataract extraction: a positive view. J Cataract Refract Surg. 2002;28(11):2060-1.

13. Javitt JC, Brenner MH, Curbow B, et al. Outcomes of cataract surgery. Improvement in visual acuity and subjective visual function after surgery in the first, second, and both eyes. Arch Ophthalmol. 1993;111(5):686-91. 